\title{
A TWO-PHASE MODEL FOR EVAPORATING SOLVENT-POLYMER MIXTURES*
}

\author{
MATTHEW G. HENNESSY ${ }^{\dagger}$, CHRISTOPHER J. W. BREWARD $^{\ddagger}$, AND \\ COLIN P. PLEASE $\ddagger$
}

\begin{abstract}
Evaporating solvent-polymer mixtures play an important role in a number of modern industrial applications. We focus on developing a two-phase model for a fluid composed of a volatile solvent and a nonvolatile polymer in a thin-film geometry. The model accounts for density differences between the phases as well as evaporation at a fluid-air interface. We use the model in one dimension to explore the interplay between evaporation and compositional buoyancy; the former promotes the growth of a polymer-rich skin at the free surface while the latter tends to pull the denser polymeric phase to the substrate. We also examine how these mechanisms influence the drying time of the film. In the limit of dilute polymer, the model can be reduced to a single nonlinear boundary value problem. The nondilute problem has a rich asymptotic structure. We find that the shortest drying times occur in the limit of strong gravitational effects due to the rapid formation of a bilayer with a polymer-rich lower layer and a solvent-rich upper layer. In addition, gravity plays a key role in inhibiting the formation of a skin and can prevent substantial increases in the drying time of the film.
\end{abstract}

Key words. evaporating films, two-phase fluids, solvent-polymer mixtures, dilute limits, skin formation

AMS subject classifications. 76T99, 35B40, 76M45

DOI. $10.1137 / 15 \mathrm{M} 1035707$

1. Introduction. Evaporating mixtures that are composed of nonvolatile polymers and a volatile solvent are present in numerous industrial applications including inkjet printing [7], painting $[14,35]$, coatings [33], the production of organic photovoltaic devices [27], and the self-assembly of polymer-based nanotechnologies [29]. The evaporation of solvent can trigger the onset of one or many physicochemical processes ranging from hydrodynamic instabilities $[1,9,20,25]$ to phase separation $[13,20,26,32]$, which can lead to nontrivial spatial distributions of polymer in the dried film $[12,23]$. The arrangement of polymers within the film, along with its surface morphology, are generally linked in some way to the quality or performance of the film $[5,6]$. This makes it tempting to try to optimize the quality of the film over different morphologies and arrangements of polymer. Realizing this goal in practice is remarkably challenging however, as it is often unclear which experimental conditions should be used to create a film with a given distribution of polymer.

The industrial relevance of evaporating solvent-polymer blends has driven theoretical studies and there is now extensive literature on the subject $[3,14,18,19,21,28$, $31,35]$. A unifying theme is the use of drift-flux models to simplify the mathematical

${ }^{*}$ Received by the editors August 18, 2015; accepted for publication (in revised form) May 19, 2016; published electronically August 30, 2016.

http://www.siam.org/journals/siap/76-4/M103570.html

Funding: This work was supported by the King Abdullah University of Science and Technology (KAUST) (Award KUK-C1-013-04).

${ }^{\dagger}$ Mathematical Institute, University of Oxford, Andrew Wiles Building, Radcliffe Observatory Quarter, Woodstock Road, Oxford, OX2 6GG, UK. Current address: Department of Chemical Engineering, Imperial College London, South Kensington Campus, London, SW7 2AZ, UK (m.hennessy@ imperial.ac.uk).

${ }^{\ddagger}$ Mathematical Institute, University of Oxford, Andrew Wiles Building, Radcliffe Observatory Quarter, Woodstock Road, Oxford, OX2 6GG, UK (breward@maths.ox.ac.uk, please@maths.ox.ac. uk). 
description of a mixture consisting of multiple chemical species with distinct physical properties, e.g., density and rheology. The key idea behind the drift-flux model is to reduce the number of governing equations by considering the mass fluxes of the individual phases but only the total momentum of the mixture [22]. For solvent-polymer systems, this generally means that the mass flux of each species is decomposed into an advective flux associated with the mean mixture velocity and a Fickian diffusive flux that is proportional to the local gradient in concentration of that species. The solvent and the polymer are assumed to have the same density, which leads to the mean mixture velocity satisfying the usual incompressibility condition. Furthermore, a single momentum balance is considered for the mixture. Consequently, the rheologies of the individual species are lumped into a composition-dependent mean viscosity associated with the mixture $[14,28]$. In some instances, drift-flux models can be simplified using lubrication theory and invoking the well-mixed approximation, whereby concentration gradients in the direction normal to the substrate are neglected due to the thinness of the fluid layer $[14,15,34]$. These models are mathematically tractable, but they have some shortcomings. For example, the polymer is generally much denser than the solvent. Furthermore, the assumption that Fick's law holds throughout the entire drying process, particularly when the solvent and polymer have similar concentrations, is questionable. Finally, well-mixed models, which are a subset of drift-flux models, neglect vertical composition gradients and are unable to capture the formation of polymer-rich skins at the surface of the fluid layer.

In this paper, we take an alternative approach to modeling evaporating solventpolymer mixtures. In particular, we formulate true multiphase models that consider mass and momentum balances for each of the constitutive components of the mixture. Although these models are more complicated than the drift-flux and well-mixed models previously described, they can easily account for density and rheological differences between the mixture components as well as capture skin formation.

Density differences between the solvent and polymer can generate flows that may play an important role during the drying process. When the polymer is denser than the solvent, buoyancy may cause the solvent to rise to the surface and the polymer to sink towards the substrate. This mode of buoyancy, which is driven by density differences in the constitutive components of the mixture, is referred to as compositional buoyancy. In contrast to the effects of compositional buoyancy, evaporation tends to drive an accumulation of polymer at the air-mixture interface. Under certain conditions [28], this accumulation of polymer will be sufficiently severe to induce the formation of a viscous skin at the free surface. From an industrial point of view, a polymer-rich skin can have detrimental consequences [3,31], as it can substantially increase the time required to dry the film. Alternatively, the high viscosity of the skin can lock-in undesirable transient film morphologies, such as those with deformed upper surfaces. If the glass transition is crossed during the drying process, the skin can buckle $[8,30]$, potentially causing it to delaminate from the underlying solvent layer, crack, or form other major defects that negatively impact the quality of the film. A pertinent question to address is, therefore, whether the opposing action of gravity can play a role in mediating the onset or effects of a polymer-rich skin.

The possibility of evaporation driving the formation of a dense layer of polymerrich fluid on top of a less dense layer of solvent-rich fluid naturally leads to the question of whether the system will be susceptible to a buoyancy-driven instability. Indeed, the numerical simulations of a two-phase fluid model by Bergantz and $\mathrm{Ni}$ [2] have shown that such layered configurations are prone to a Rayleigh-Taylor instability. As a result, the denser upper layer decomposes into plumes that sink towards the substrate. 


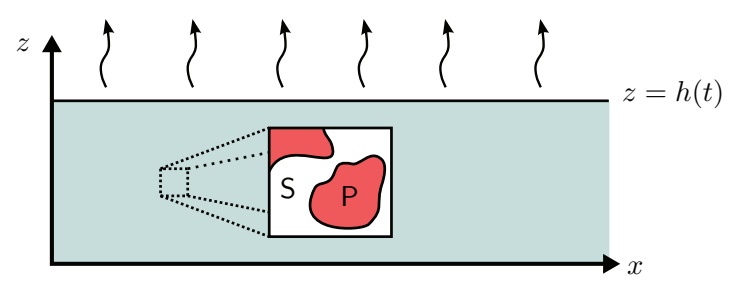

FIG. 1. We consider the macroscopic dynamics of a binary mixture composed of solvent $\mathbf{S}$ and polymer $\mathrm{P}$. The mixture forms a layer on top of a nonporous substrate. Solvent is free to evaporate at the surface of the layer.

When analyzing the onset of convection in the evaporating toluene-polystyrene mixtures considered by Bassou and Rharbi [1], Hennessy and Münch [18] concluded that compositional-Marangoni instabilities are too weak by themselves to initiate convection, and proposed that compositional buoyancy may also play an important role.

The purpose of this paper is to formulate a two-phase model to describe an evaporating solvent-polymer mixture and then use it to investigate the interplay between gravity, evaporation, and skin formation. In section 2, we give an overview of the system under consideration and our primary modeling assumptions. We then present a macroscopic model, based on a rigorous averaging of a microscopic model, for an evaporating two-phase solvent-polymer mixture. In section 3 , the dynamics that occur in the limit of a dilute polymeric phase are explored. We then relax this assumption in section 4 and consider the dynamics of a nondilute mixture. We determine the conditions under which a polymer-rich skin forms and explore how the drying time is influenced by gravity and skin formation. Finally, in section 5 we will discuss the results and draw conclusions.

2. Formulation of the model. In this paper, we will consider the behavior of an immiscible mixture of polymer and solvent in a layer on a horizontal substrate, as shown in Figure 1. The substrate is impermeable while the free surface allows for evaporation of solvent. Surface tension at the air-mixture interface is assumed to be sufficiently strong that any surface deflections are negligibly small. Thus, we suppose that the air-mixture interface is always flat. At the macroscopic level, the system is assumed to be well mixed initially. The key competing mechanisms that drive the flow are gravity, due to the polymer being more dense than the solvent, and evaporation; our aim is to consider the macroscopic interplay between the two.

Our macroscale model is derived through a systematic averaging of appropriate equations describing the microscopic properties of the mixture. We do not present the averaging here. Rather, we give the key facets of the recipe and refer the reader to Drew [10] and Drew and Passman [11] for the general methodology or Hennessy [17] for the specific details.

The derivation of the macroscopic model begins with a consideration of the microscopic dynamics. Descriptions of the bulk properties of each phase along with the physics and chemistry occurring at phase boundaries are fundamental. We treat the two phases as immiscible liquids and assume that the mixture is composed of many microscopic domains that are pure in polymer or solvent, as illustrated in Figure 1. Each domain is taken to be a volume of slowly flowing viscous liquid whose motion can be described by the Stokes equations. Our assumption of immiscibility implies that no material of one phase passes through the interface formed with the other; thus, kinematic conditions hold at solvent-polymer boundaries. At these boundaries, 
we also assume that there is no slip between the phases and that stress is continuous. The overlying air is treated as inviscid and at constant pressure.

In order to make the transition from the microscale to the macroscale, we average the microscopic model and invoke the following assumptions: the mean density and viscosity of each phase is constant; the drag between the solvent and polymer, which is a manifestation of the no-slip boundary condition, is proportional to the difference in velocity of the two phases; the surface tension and normal components of the deviatoric stresses at microscopic boundaries are negligible; and, for each phase, the mean pressure in the bulk is equal to the pressure at the phase boundaries. These last two conditions imply that the mean pressures of all of the phases are equal to each other; therefore, the number of pressure variables in the macroscopic model is reduced to one.

We now present the macroscopic governing equations. For simplicity, we will restrict our attention to the one-dimensional problem, although extending the model to multiple spatial dimensions is straightforward. The one-dimensional equations for the volume fractions of polymer and solvent, $\alpha_{p}$ and $\alpha_{s}$, respectively, the vertical velocities of polymer and solvent, $w_{p}$ and $w_{s}$, and the pressure $p$ are

$$
\begin{aligned}
\frac{\partial \alpha_{p}}{\partial t}+\frac{\partial}{\partial z}\left(\alpha_{p} w_{p}\right) & =0, \\
\frac{\partial}{\partial z}\left[\left(1-\alpha_{p}\right) w_{s}+\alpha_{p} w_{p}\right] & =0, \\
-\alpha_{p} \frac{\partial p}{\partial z}+2 \mu_{p} \frac{\partial}{\partial z}\left(\alpha_{p} \frac{\partial w_{p}}{\partial z}\right)-\alpha_{p} \rho_{p} g+c_{s p} \alpha_{p}\left(1-\alpha_{p}\right)\left(w_{s}-w_{p}\right) & =0, \\
-\frac{\partial p}{\partial z}+2 \frac{\partial}{\partial z}\left[\mu_{s}\left(1-\alpha_{p}\right) \frac{\partial w_{s}}{\partial z}+\mu_{p} \alpha_{p} \frac{\partial w_{p}}{\partial z}\right]-\left[\left(1-\alpha_{p}\right) \rho_{s}+\alpha_{p} \rho_{p}\right] g & =0, \\
\alpha_{p}+\alpha_{s} & =1,
\end{aligned}
$$

where $t$ is time and $z$ is the upwards-pointing vertical coordinate. Here, $\mu_{j}$ and $\rho_{j}$ correspond to the mean kinematic viscosity and density of phase $j=s, p$, respectively; $g$ is the gravitational acceleration; and $c_{s p}$ is the drag coefficient between solvent and polymer.

Equations (2.1a) and (2.1c) represent the conservation of mass and momentum for the polymeric phase; $(2.1 \mathrm{~b})$ and $(2.1 \mathrm{~d})$ express conservation of mass and momentum for the mixture; and (2.1e) enforces the requirement that each point in space is occupied by the solvent and/or the polymer, i.e., no voids form in the mixture. We note in passing that conservation equations for the solvent can easily be obtained by subtracting the equations for the polymer from those for the mixture and then using (2.1e) to replace $\alpha_{p}$ with $\alpha_{s}$.

The no-flow boundary conditions along the substrate are

$$
w_{j}=0, \quad z=0, \quad j=s, p .
$$

At the flat free surface where the solvent can evaporate, conservation of mass gives the kinematic conditions

$$
\begin{array}{ll}
w_{s}-\frac{\mathrm{d} h}{\mathrm{~d} t}=E\left(1-\alpha_{p}\right), & z=h(t), \\
w_{p}-\frac{\mathrm{d} h}{\mathrm{~d} t}=0, & z=h(t),
\end{array}
$$

where $E$ is the evaporation velocity, which is defined as the rate at which a static layer of pure solvent thins; note that $w_{s} \equiv 0$ and hence $\mathrm{d} h / \mathrm{d} t=-E$ when $\alpha_{p} \equiv 0$. Moreover, $\rho_{s} E$ corresponds to a mass transfer coefficient. 
The continuity of normal stress at the flat free surface merits a brief discussion. As there is only a single pressure variable in our model, corresponding to that of the mixture, we require that the normal stress of the mixture is continuous across the air-mixture interface. This yields a single condition for the normal stress given by

$$
-p+2\left[\mu_{s}\left(1-\alpha_{p}\right) \frac{\partial w_{s}}{\partial z}+\mu_{p} \alpha_{p} \frac{\partial w_{p}}{\partial z}\right]=-p_{a}, \quad z=h(t),
$$

where $p_{a}$ denotes the constant pressure of the air.

An equation governing the free surface can be obtained by integrating $(2.1 \mathrm{~b})$ over the thickness of the fluid layer and using the no-flow conditions $w_{s}=w_{p}=0$ at $z=0$ as well as the kinematic conditions in $(2.1 \mathrm{~g})$ and $(2.1 \mathrm{~h})$. We find that the free surface evolves according to

$$
\frac{\mathrm{d} h}{\mathrm{~d} t}=-E\left[1-\alpha_{p}(h(t), t)\right]^{2} .
$$

If we multiply $(2.1 \mathrm{j})$ by $\rho_{s}$, its right-hand side can be interpreted as the product of the evaporative mass flux, $\rho_{s} E\left(1-\alpha_{p}\right)$, and the fraction of the free surface that is occupied by the solvent, $\left(1-\alpha_{p}\right)$. We emphasize that $(2.1 \mathrm{j})$ is a consequence of applying a global mass balance to the two-phase model; it is not an additional constitutive relation.

We assume that, on a macroscopic level, the polymer is initially uniformly distributed throughout the solvent. Therefore, initial conditions for this problem are given by

$$
\alpha_{p}(z, 0)=A, \quad h(0)=H .
$$

2.1. Nondimensionalization. The macroscopic two-phase model is scaled in a manner that makes the role of buoyancy most apparent. We take

$$
z=H z^{\prime}, \quad t=(H / E) t^{\prime}, \quad p=\left(\mu_{p} E / H\right) p^{\prime}-\rho_{p} g H z^{\prime}+p_{a}, \quad w_{i}=E w_{i}^{\prime} .
$$

The resulting nondimensional equations in the bulk are (upon dropping the primes)

$$
\begin{aligned}
\frac{\partial \alpha_{p}}{\partial t}+\frac{\partial}{\partial z}\left(\alpha_{p} w_{p}\right) & =0, \\
\frac{\partial}{\partial z}\left[\left(1-\alpha_{p}\right) w_{s}+\alpha_{p} w_{p}\right] & =0, \\
-\alpha_{p} \frac{\partial p}{\partial z}+2 \frac{\partial}{\partial z}\left(\alpha_{p} \frac{\partial w_{p}}{\partial z}\right)+D \alpha_{p}\left(1-\alpha_{p}\right)\left(w_{s}-w_{p}\right) & =0, \\
-\frac{\partial p}{\partial z}+2 \frac{\partial}{\partial z}\left[\mu\left(1-\alpha_{p}\right) \frac{\partial w_{s}}{\partial z}+\alpha_{p} \frac{\partial w_{p}}{\partial z}\right]+R\left(1-\alpha_{p}\right) & =0,
\end{aligned}
$$

where

$$
R=\frac{\left(\rho_{p}-\rho_{s}\right) g H^{2}}{\mu_{p} E}, \quad D=\frac{c_{s p} H^{2}}{\mu_{p}}, \quad \mu=\frac{\mu_{s}}{\mu_{p}}
$$

define a compositional Rayleigh number, the effective drag coefficient, and the relative kinematic viscosity. The Rayleigh number $R$ characterizes the relative importance of buoyancy-driven flow, which results from differences in the solvent and polymer densities. The effective drag coefficient describes the strength of drag forces to viscous stress. In section 4.1 we will show that when $R, D \ll 1$, the polymer volume fraction 
remains uniform in space. Thus, the well-mixed approximation that is encountered in the literature $[14,15,34]$ can be obtained from the two-phase model in the limit of weak drag and weak gravitational effects. Since our model is based on the premise that the two fluids are immiscible, additional physics are required if the drift-flux models, which account for mixing of two miscible phases due to diffusion, are to be recovered. In Appendix A we outline the relationship between drift-flux models and the two-phase model presented here.

The nondimensional boundary conditions at the substrate are given by

$$
w_{j}=0, \quad z=0, \quad j=s, p,
$$

and those at the free surface can be written as

$$
\begin{aligned}
w_{s}-\frac{\mathrm{d} h}{\mathrm{~d} t} & =1-\alpha_{p}, & & z=h(t), \\
w_{p}-\frac{\mathrm{d} h}{\mathrm{~d} t} & =0, & & z=h(t), \\
-p+2\left[\mu\left(1-\alpha_{p}\right) \frac{\partial w_{s}}{\partial z}+\alpha_{p} \frac{\partial w_{p}}{\partial z}\right] & =-G h, & & z=h(t),
\end{aligned}
$$

where $G$ is a Galileo number defined by

$$
G=\frac{\rho_{p} g H^{2}}{\mu_{p} E}
$$

The nondimensional equation for the free surface reads

$$
\frac{\mathrm{d} h}{\mathrm{~d} t}=-\left[1-\alpha_{p}(h(t), t)\right]^{2} .
$$

The initial conditions are given by $\alpha_{p}(z, 0)=A$ and $h(0)=1$.

Due to the one-dimensional nature of the model, it is possible to simplify the problem by eliminating the pressure and the solvent velocity from the governing equations. By integrating the incompressibility condition (2.3b) and applying the boundary conditions on the substrate, we find that the solvent velocity can be written as

$$
w_{s}=-\frac{\alpha_{p}}{1-\alpha_{p}} w_{p}
$$

It is straightforward to verify that the solution for the solvent velocity (2.9) also satisfies the kinematic boundary conditions at the free surface given by (2.6a) and (2.6b). By combining (2.3c) and (2.3d) to eliminate the pressure gradient and by using (2.9) to remove the solvent velocity from the problem, a nonlinear differential equation for the polymer velocity emerges. Thus, the one-dimensional bulk equations can be written solely in terms of variables associated with the polymer:

$$
\begin{aligned}
\frac{\partial \alpha_{p}}{\partial t}+\frac{\partial}{\partial z}\left(\alpha_{p} w_{p}\right) & =0, \\
2\left(1-\alpha_{p}\right) \frac{\partial}{\partial z}\left(\alpha_{p} \frac{\partial w_{p}}{\partial z}\right)+2 \mu \alpha_{p} \frac{\partial}{\partial z}\left[\left(1-\alpha_{p}\right) \frac{\partial}{\partial z}\left(\frac{\alpha_{p} w_{p}}{1-\alpha_{p}}\right)\right] & \\
-D \alpha_{p} w_{p}-R \alpha_{p}\left(1-\alpha_{p}\right) & =0 .
\end{aligned}
$$

Copyright (c) by SIAM. Unauthorized reproduction of this article is prohibited. 
The film height satisfies

$$
\frac{\mathrm{d} h}{\mathrm{~d} t}=-\left[1-\alpha_{p}(h(t), t)\right]^{2} .
$$

Initial conditions are $\alpha_{p}(z, 0)=A$ and $h(0)=1$. Boundary conditions for the polymer velocity are given by

$$
\begin{array}{ll}
w_{p}=0, & z=0, \\
w_{p}=-\left(1-\alpha_{p}\right)^{2}, & z=h(t) .
\end{array}
$$

The kinematic condition at the free surface $(2.10 \mathrm{e})$ has been simplified by replacing $\mathrm{d} h / \mathrm{d} t$ using (2.10c).

In summary, we will solve the two-phase model in (2.10) for the volume fraction and velocity of polymer, $\alpha_{p}$ and $w_{p}$, respectively, and the film thickness $h$. The volume fraction and velocity of solvent can be immediately deduced from (2.1e) and (2.9). Finally, the mixture pressure $p$ can be calculated by integrating the mixture momentum balance $(2.3 \mathrm{~d})$ and using the boundary condition $(2.6 \mathrm{~b})$.

Our analysis of (2.10) is split into two sections. In section 3, the behavior of a mixture with a dilute polymeric phase is examined in various parameter regimes by taking $A \rightarrow 0$ in (2.10). The model for a dilute polymer eventually breaks down when the accumulation of polymer becomes sufficiently large. Thus, we return to the original model for a nondilute polymer in section 4 .

3. The dilute-polymer model. It is often the case in experiments that the initial concentration of polymer is low, ranging from a few percent up to $20 \%$. Thus, it is reasonable to consider a dilute-polymer limit by taking $A \searrow 0$. Although this limit will lead to substantial simplifications in the governing equations, the resulting model will only be valid until a critical time is reached, after which the polymer concentration becomes sufficiently large that it can no longer be treated as dilute. A full examination could explore linking the dilute and nondilute models using matched asymptotic expansions; however, this is not something that we consider here.

The dilute-polymer model is formulated by writing $\alpha=A \alpha^{(0)}, w_{p}=w_{p}^{(0)}$, and $h=h^{(0)}$ in (2.10) and then taking $A \searrow 0$. From (2.10c) it immediately follows that the leading-order solution for the film thickness is given by

$$
h^{(0)}(t)=1-t .
$$

Thus, in the dilute limit, the film thins at a constant rate due to the evaporative mass flux being independent of the surface composition at leading order, and the film thickness decreases to zero in finite time. From (3.1), we see that the dilute model will break down as $t \nearrow 1$; however, it may happen before this time if the scaled polymer volume fraction $\alpha_{p}^{(0)}$ becomes of size $O\left(A^{-1}\right)$. Assuming the dilute approximation is valid, the problem for the polymer can be written as

$$
\begin{aligned}
\frac{\partial \alpha_{p}^{(0)}}{\partial t}+\frac{\partial}{\partial z}\left(\alpha_{p}^{(0)} w_{p}^{(0)}\right) & =0 \\
2 \frac{\partial}{\partial z}\left(\alpha_{p}^{(0)} \frac{\partial w_{p}^{(0)}}{\partial z}\right)-D \alpha_{p}^{(0)} w_{p}^{(0)}-R \alpha_{p}^{(0)} & =0
\end{aligned}
$$


with boundary and initial conditions

$$
\begin{array}{ll}
w_{p}^{(0)}=0, & z=0, \\
w_{p}^{(0)}=-1, & z=1-t, \\
\alpha_{p}^{(0)}=1, & t=0 .
\end{array}
$$

The bulk equations for the solvent in the dilute-polymer limit are given by the onedimensional incompressible Stokes equations

$$
\begin{aligned}
-\frac{\partial p^{(0)}}{\partial z}+2 \mu \frac{\partial^{2} w_{s}^{(0)}}{\partial z^{2}}+R & =0, \\
\frac{\partial w_{s}^{(0)}}{\partial z} & =0 .
\end{aligned}
$$

The dilute-polymer model bears a marked resemblance to the drift-flux and well-mixed models, which use the incompressible Stokes equations to describe the momentum balance of the mixture (see Appendix A). However, the dilute-polymer model (3.2) differs substantially from the other two models because it considers a momentum balance for the polymer instead of prescribing an assumed form of the mass flux.

Despite the simple appearance of the dilute model given by (3.2), it is remarkably difficult to solve analytically. However, by rewriting this model in terms of a Lagrangian coordinate, the governing equations can be significantly simplified and analytical progress can be made on the problem. We now outline the simplification procedure.

3.1. Reduction to a single differential equation. We begin by introducing a Lagrangian coordinate $s \in[0,1]$ that is fixed to material points in the polymer phase. It will be assumed that the Eulerian coordinate $z$ and the Lagrangian coordinate $s$ are identical at $t=0$ and that $s=0$ and $s=1$ correspond to material points at the substrate and the free surface, respectively. We then reformulate the problem for the Eulerian volume fraction $\alpha_{p}^{(0)}(z, t)$ and the velocity $w_{p}^{(0)}(z, t)$ given by $(3.2)$ in terms of the Lagrangian volume fraction $a_{p}^{(0)}(s, t)$ and the Eulerian coordinate $z(s, t)$. We will show that the Lagrangian formulation of conservation of polymer (3.2a) leads to a simple expression for $a_{p}^{(0)}(s, t)$, which can be used to derive a boundary value problem for $z(s, t)$.

We now apply the method of characteristics to (3.2a) by writing

$$
\begin{aligned}
\frac{\partial z}{\partial t} & =w_{p}^{(0)}, \\
\frac{\partial a_{p}^{(0)}}{\partial t} & =-a_{p}^{(0)} \frac{\partial w_{p}^{(0)}}{\partial z} .
\end{aligned}
$$

The Lagrangian coordinate $s$ is used to parameterize different characteristic curves. Initial and boundary conditions for $(3.4)$ are given by $z(s, 0)=s, a_{p}^{(0)}(s, 0)=1$, $z(0, t)=0$, and $z(1, t)=1-t$. From the chain rule we find that

$$
\frac{\partial}{\partial z}=\left(\frac{\partial z}{\partial s}\right)^{-1} \frac{\partial}{\partial s}
$$

Thus, we can write $\partial w_{p}^{(0)} / \partial z=\left(\partial w_{p}^{(0)} / \partial s\right) /(\partial z / \partial s)$ and, by using (3.4a), we have

$$
\frac{\partial w_{p}^{(0)}}{\partial z}=\frac{\partial^{2} z /(\partial s \partial t)}{\partial z / \partial s}
$$


By substituting (3.6) into (3.4b), it can be shown that

$$
a_{p}^{(0)} \frac{\partial z}{\partial s}=1,
$$

which expresses conservation of mass in Lagrangian coordinates. Equations (3.5) and (3.7) provide a means of transforming $z$ derivatives via

$$
\frac{\partial}{\partial z}=a_{p}^{(0)} \frac{\partial}{\partial s}
$$

Using (3.8) along with (3.4a) and (3.4b) in the momentum equation (3.2b) leads to significant simplifications, giving

$$
2 \frac{\partial}{\partial t}\left(\frac{\partial a_{p}^{(0)}}{\partial s}\right)+D \frac{\partial z}{\partial t}+R=0 .
$$

By integrating (3.9) with respect to $t$, differentiating (3.7) with respect to $s$, and combining the results, a second-order nonlinear boundary value problem for $z$ is obtained. This is given by

$$
2 \frac{\partial^{2} z}{\partial s^{2}}+[D(s-z)-R t]\left(\frac{\partial z}{\partial s}\right)^{2}=0
$$

which has boundary conditions

$$
z(0, t)=0, \quad z(1, t)=1-t .
$$

The initial condition for (3.2) has been incorporated into (3.10) through (3.7) and the time variable $t$ now acts as a parameter. By solving (3.10) for $z$, the volume fraction and velocity of polymer can be obtained directly from (3.7) and (3.4a), respectively. This process gives a parametric solution in terms of the Lagrangian coordinate $s$. When $\partial z / \partial s \neq 0$, the mapping between $z$ and $s$ is one-to-one, implying that no shocks occur, and the solution, in principle, can be written in terms of the original Eulerian coordinate $z$.

The product $R t$ appearing in (3.10) is particularly interesting, as it implies that $1 / R$ defines a second time scale corresponding to the time required for the denser phase to settle on the substrate. The cases $R \ll 1$ and $R \gg 1$ are associated with situations where the settling process is slow and fast compared to evaporation, respectively.

We will now examine the behavior of the dilute-polymer model by seeking approximate solutions to (3.10) that are valid in the limits of (i) weak drag and (ii) as the final time is approached, i.e., when $t \nearrow 1$. These regimes, and the dynamics that occur in each of them, are summarized in Figure 2.

3.2. Solution of the dilute model with weak drag. In the limit of weak drag, $D \ll 1$, the problem for $z$ given by (3.10) reduces to

$$
\frac{\partial^{2} z}{\partial s^{2}}-\frac{R t}{2}\left(\frac{\partial z}{\partial s}\right)^{2}=0
$$

with $z(0, t)=0$ and $z(1, t)=1-t$. Equation (3.12) has the analytical solution

$$
z(s, t)=-\frac{2}{R t} \log (s[\exp \{-R t(1-t) / 2\}-1]+1) .
$$

Copyright (C) by SIAM. Unauthorized reproduction of this article is prohibited. 


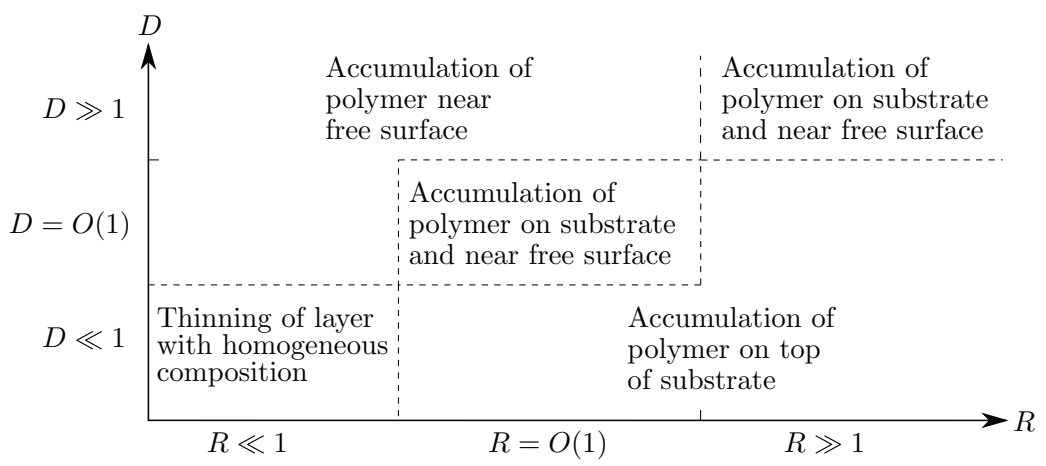

FIG. 2. Dynamics that occur in the dilute model (3.2) for various values of the Rayleigh number $R$ and the effective drag coefficient $D$. Analytical solutions can be found when $R, D \ll 1$ and $D \ll 1$. The behavior in the other regimes is deduced by examining the model as it approaches the final time, $t \nearrow 1$.

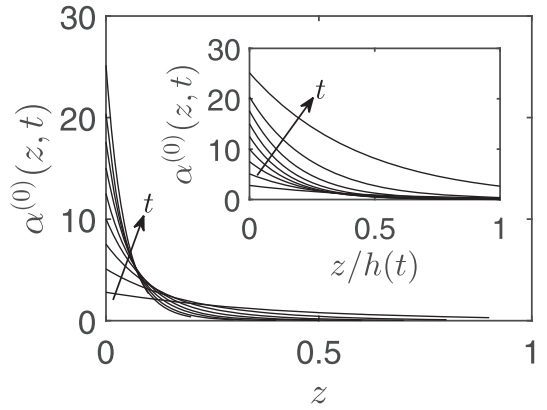

(a)

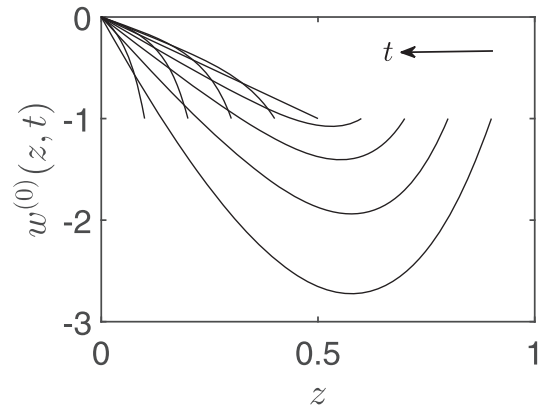

(b)

FIG. 3. Evolution of the leading-order polymer volume fraction (a) and velocity (b) as computed from the dilute-polymer model (3.2) in the limit of negligible drag. Arrows represent the direction of increasing time. For clarity, the inset of panel (a) shows the volume fraction on a fixed spatial domain. Gravitational effects lead to a strong accumulation of polymer near the substrate. Solutions are shown at times $t=0.1$ to $t=0.9$ at increments of $\Delta t=0.1$. In addition, we take $R=50$ and $D=0$.

The volume fraction of polymer, written in terms of Eulerian variables, is given by

$$
\alpha_{p}^{(0)}(z, t)=\frac{R t}{2} \frac{\exp (-R t z / 2)}{1-\exp [-R t(1-t) / 2]} .
$$

A complicated closed-form expression for the velocity can be computed from (3.13) using the fact that $w_{p}^{(0)}=\partial z / \partial t$; however, we do not present this here.

The solution given in (3.14) implies that for each time $t$, the volume fraction decays exponentially with the distance from the substrate, corresponding to an accumulation of polymer near the bottom of the fluid layer. Indeed, this behavior is clearly seen in Figure 3(a), which shows the evolution of the polymer volume fraction (3.14) when $R=50$ and $D=0$. The parabolic nature of the corresponding velocity profile seen in Figure 3(b) at early times reflects the fact that strong gravitational forces cause the polymer to sink faster than the rate at which the film thins. At later times, the velocity becomes monotonic as the polymer becomes packed against the bottom surface. 
For $R \ll 1$, the solution for the volume fraction (3.14) can be expanded as $\alpha_{p}^{(0)}(z, t)=(1-t)^{-1}+O(R)$. Thus, in the limit of weak drag and gravity, the composition of the mixture remains spatially uniform.

3.3. Behavior of the dilute model near the final time. We now consider (3.10) in the limit as $t \nearrow 1$ when both gravity and drag are nonnegligible. This will provide a means of exploring the final behavior of solutions to the dilute model (3.2). From this, we can determine the regions of parameter space associated with gravityand drag-dominated systems.

We begin by introducing a new time variable $\tilde{t}$ such that $t=1-\delta \tilde{t}$ with $\delta \ll 1$ and $\tilde{t}=O(1)$. The boundary conditions (3.11) suggest that $z=O(\delta)$ as $t \nearrow 1$; thus, we write $z(s, t)=\delta \tilde{t} \tilde{z}(s, \tilde{t})$. The governing equation for $\tilde{z}$ is given by

$$
\frac{\partial^{2} \tilde{z}}{\partial s^{2}}+\frac{\delta \tilde{t}}{2}[D(s-\delta \tilde{t} \tilde{z})-R(1-\delta \tilde{t})]\left(\frac{\partial \tilde{z}}{\partial s}\right)^{2}=0
$$

and the corresponding boundary conditions are $\tilde{z}(0, \tilde{t})=0$ and $\tilde{z}(1, \tilde{t})=1$. A matching condition (in time) is not required for this problem since (3.10), and hence (3.15), are the result of integrating the dilute model (3.2) in time along the characteristic directions. We now compute asymptotic solutions to (3.15) when the effective strengths of gravity and drag are moderate and strong, corresponding to $R, D=O(1)$ and $R, D=O\left(\delta^{-1}\right)$, respectively.

If $R, D=O(1)$, we can use a regular asymptotic expansion in powers of $\delta$ to obtain an approximate solution of (3.15). The first two terms in the solution for the Eulerian coordinate, $z$, and the Lagrangian volume fraction, $a_{p}^{(0)}$, written as functions of the original time variable $t$, are

$$
\begin{aligned}
z(s, t) & =(1-t) s+\frac{(1-t)^{2}}{4}\left[\frac{D}{3}(s+1)-R\right] s(1-s), \\
a_{p}^{(0)}(s, t) & =(1-t)^{-1}-\frac{1}{4}\left[D\left(\frac{1}{3}-s^{2}\right)+R(2 s-1)\right] .
\end{aligned}
$$

The distribution of polymer, therefore, remains roughly spatially uniform during the drying process, with small variations caused by moderate effects of gravity and drag. Gravity tends to drive an accumulation of polymer near the substrate, whereas drag leads to a buildup of polymer near the free surface. We define gravity- and dragdominated systems as those in which the greatest accumulation of polymer occurs at the substrate and free surface, respectively.

By analyzing (3.17), we find that when $R / D>1$, the volume fraction is monotonically decreasing on the interval $0 \leq s \leq 1$, implying that the system is gravity dominated. When $R / D<1$, the volume fraction has an interior minimum at $s=R / D$; the system remains gravity dominated when $1 / 2<R / D<1$ but it becomes drag dominated when $R / D<1 / 2$. In both of these subcases, the interior minimum at $s=R / D$ corresponds to the Eulerian coordinate $z_{c}(t) \simeq(1-t) R / D$.

The situation when the effects of drag and gravity are strong can be analyzed by considering the distinguished limit $R=\delta^{-1} \tilde{R}$ and $D=\delta^{-1} \tilde{D}$, with $\tilde{R}, \tilde{D}=O(1)$, in (3.15). The leading-order problem is given by

$$
\frac{\partial^{2} \tilde{z}}{\partial s^{2}}+\frac{\tilde{t}}{2}(\tilde{D} s-\tilde{R})\left(\frac{\partial \tilde{z}}{\partial s}\right)^{2}=0
$$

Copyright $@$ by SIAM. Unauthorized reproduction of this article is prohibited. 
with $\tilde{z}(0, \tilde{t})=0$ and $\tilde{z}(1, \tilde{t})=1$. The solution to (3.18) can be found analytically and written in terms of the original variables to obtain

$$
z(s, t)=\frac{4}{c(t)} f(s ; c(t)), \quad f(s ; c)=\arctan \left(\frac{D s-R}{c}\right)+\arctan \left(\frac{R}{c}\right),
$$

where $c=c(t)$ solves

$$
(1-t) c(t)=4 f(1 ; c(t))
$$

Using (3.19), the leading-order solution for the volume fraction $a_{p}^{(0)}$ is found to be a quadratic function of the Lagrangian coordinate:

$$
a_{p}^{(0)}(s, t)=\frac{1}{4 D}\left[c(t)^{2}+(D s-R)^{2}\right] .
$$

When $R / D<1$, the volume fraction in (3.21) has an interior minimum at $s=$ $R / D<1$, corresponding to the Eulerian coordinate

$$
z_{c}(t)=\frac{4}{c(t)} \arctan \left(\frac{R}{c(t)}\right)
$$

However, when $R / D>1$, the volume fraction is monotonically decreasing. Comparing the volume fraction at the surface and substrate shows that the system is gravity dominated when $R>D / 2$ and drag dominated when $R<D / 2$. Thus, the characterization of the dominant regimes when $R, D \gg 1$ is the same as the case $R, D=O(1)$ analyzed previously. A parametric solution for the velocity can be obtained via the relation $w_{p}^{(0)}=\partial z / \partial t$; however, the result is sufficiently complicated that no insight is gained by writing it here.

A comparison of the asymptotic expressions for the volume fraction given by (3.19)-(3.21) and the velocity with numerical solutions is shown in Figure 4. The numerical approximation is obtained by solving the nonlinear boundary value problem in (3.10) using a Chebyshev spectral method. Three cases are shown in this figure: $R<D / 2, D / 2<R<D$, and $R>D$, corresponding to the drag- and gravitydominated behaviors discussed previously. The asymptotic and numerical approximations are in excellent agreement with each other, thus validating our characterization of the key parameter regimes.

4. The nondilute problem. For the remainder of the paper, we focus on the dynamics that occur when the polymer phase is nondilute by assuming that $A=O(1)$. Unlike in the dilute case, switching to a Lagrangian coordinate does not lead to any simplifications in (2.10). This is rather intuitive and it reflects the fact there are two possible Lagrangian coordinates in the nondilute problem: one for each phase. Therefore, switching to one of these coordinates simplifies the terms for the corresponding phase in (2.10), but this comes at a cost of increasing the complexity of the terms associated with the other phase. Thus, our analysis of the nondilute problem given by (2.10) is based on Eulerian coordinates.

In general, the nondilute problem must be solved numerically. We use an explicit scheme that decouples the mass transport and hydrodynamic problems. That is, for a given polymer volume fraction and film thickness, the polymer velocity is obtained by solving a linear system of equations. Using the newly computed velocity, the volume fraction and the film thickness are updated, and the process repeats. Further details 


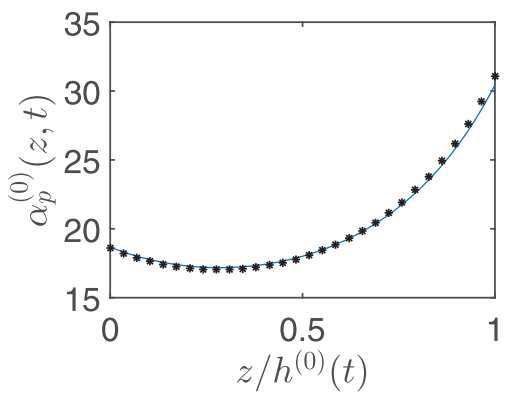

(a) $R<D / 2$

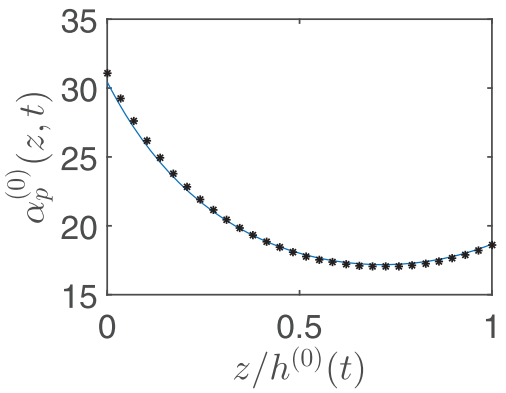

(c) $D / 2<R<D$

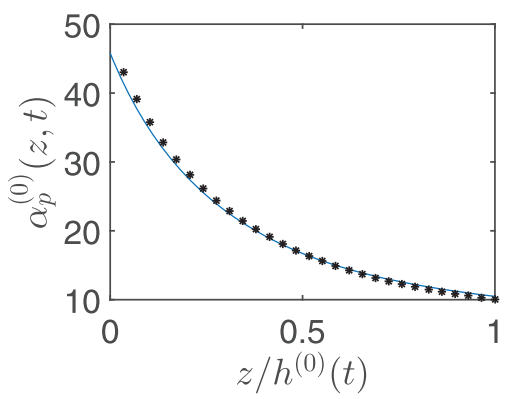

(e) $D<R$

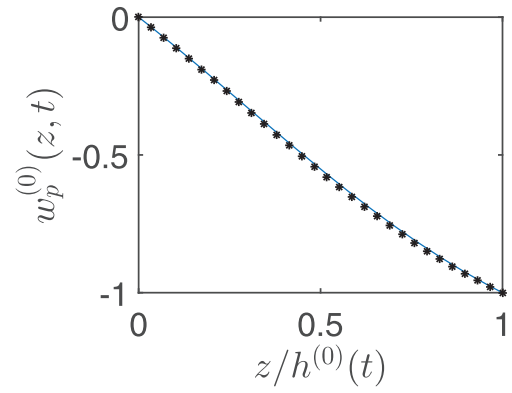

(b) $R<D / 2$

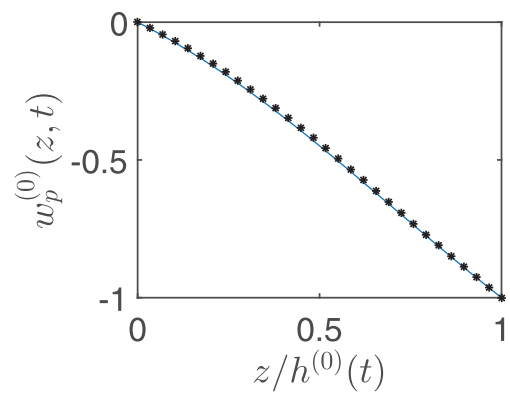

(d) $D / 2<R<D$

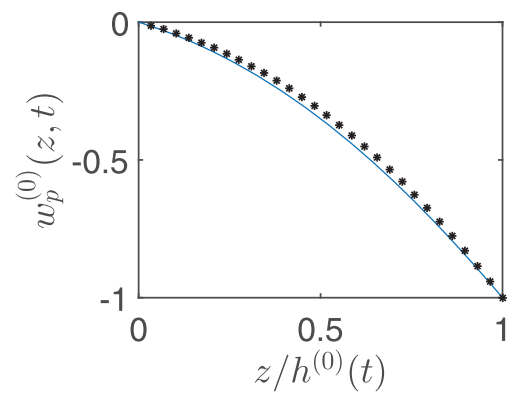

(f) $D<R$

FIG. 4. Comparison of the asymptotic solution (symbols) as $t \rightarrow 1$ with numerical solutions (lines) to (3.10) when both $R$ and $D$ are large. The polymer volume fractions and corresponding velocities are shown in the top and bottom rows, respectively. Parameter values are $t=0.95$, $D=100$, and $R=25,75$, and 125 for panels (a)-(b), (c)-(d), and (e)-(f), respectively. Panels (a) and (b) corresponds to the drag-dominated region of parameter space given by $R<D / 2$. Panels (c)-(f) represent the gravity-dominated behavior that occurs when $R>D / 2$. This can be subdivided into two regimes. If $D / 2<R<D$, the volume fraction has an interior minimum and there is some accumulation of polymer near the surface (c). If $R>D$, gravity is so strong that the volume fraction decreases monotonically (e).

of the numerical method, including the equations that are solved at each time step, are given in Appendix B. Spatial derivatives are approximated with finite-difference formulas and the MATLAB ode45 function is used for time stepping.

We now analyze the nondilute model given by (2.10) in several limiting cases using asymptotic and numerical methods. The various cases we consider are summarized in Figure 5. 


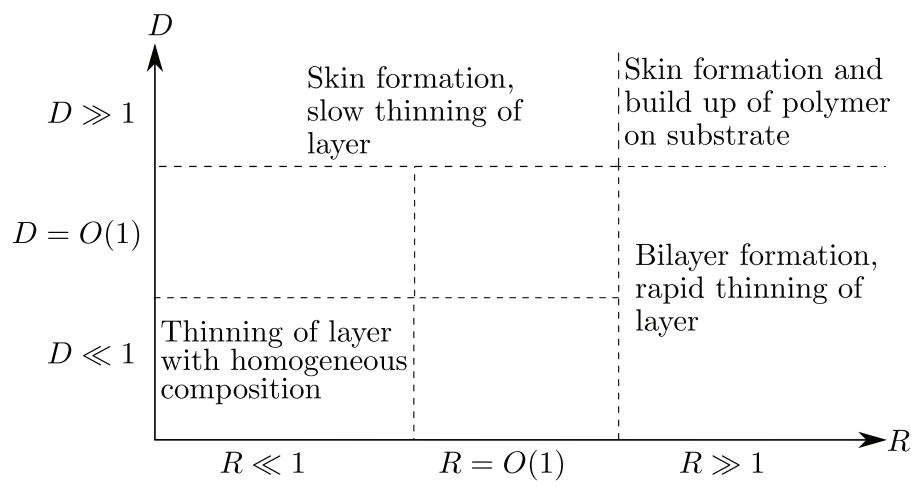

FIG. 5. Dynamics that occur in the nondilute model (2.10) for various values of the Rayleigh number $R$ and the effective drag coefficient D. Analytical solutions can be found when $R, D \ll 1$. In all other cases, the dynamics are deduced by asymptotic analyses and numerical simulations.

4.1. Weak drag and weak gravity. We first consider the simple case when both gravity and drag are weak; therefore, we set $R=D=0$ in the governing equations (2.10). The analysis of the dilute model in the regime of weak gravity and drag (see section 3.2) revealed that the volume fraction remains spatially uniform during the drying process. This motivates an ansatz for the nondilute model in this parameter regime given by $\alpha_{p}(z, t)=\alpha_{p}(t)$. Substituting this ansatz into the bulk equations (2.10a) $-(2.10 \mathrm{~b})$ results in a substantially simplified system given by

$$
\begin{aligned}
\frac{\mathrm{d} \alpha_{p}}{\mathrm{~d} t}+\alpha_{p} \frac{\partial w_{p}}{\partial z} & =0, \\
\frac{\partial^{2} w_{p}}{\partial z^{2}} & =0 .
\end{aligned}
$$

By solving (4.1b) and imposing the boundary conditions $w_{p}(0, t)=0$ and $w_{p}(h(t), t)=$ $\mathrm{d} h / \mathrm{d} t$, we find that $w_{p}=(\mathrm{d} h / \mathrm{d} t)(z / h)$. Inserting this expression for $w_{p}$ into (4.1a) and integrating in time gives $\alpha_{p}(t)=A / h(t)$. The solution for $\alpha_{p}$ can be used in the evolution equation for the film thickness $(2.10 \mathrm{c})$ to derive a nonlinear initial value problem

$$
\frac{\mathrm{d} h}{\mathrm{~d} t}=-\left(1-\frac{A}{h}\right)^{2}, \quad h(0)=1,
$$

which has an implicit solution given by

$$
h(t)-1+A^{2}\left(\frac{1}{1-A}-\frac{1}{A-h(t)}\right)+2 A \log \left(\frac{h(t)-A}{1-A}\right)=-t .
$$

As $t \rightarrow \infty$, the thickness $h \rightarrow A$, corresponding to a film that is composed of only polymer. No skin forms because the concentration of polymer remains uniform as the film dries.

Comparisons of the implicit solution for the film thickness given in (4.3) with numerical solutions are displayed in Figure 6 for two combinations of $A, R$, and $D$, with $\mu=1$. When the initial volume fraction is small (panel (a)), the implicit solution shows excellent agreement with the numerical solution despite $R$ and $D$ being $O(1)$ in size. For larger initial volume fractions (panel (b)), the implicit solution still gives a good approximation of the film thickness; however, this is only the case for smaller values of $R$ and $D$. 


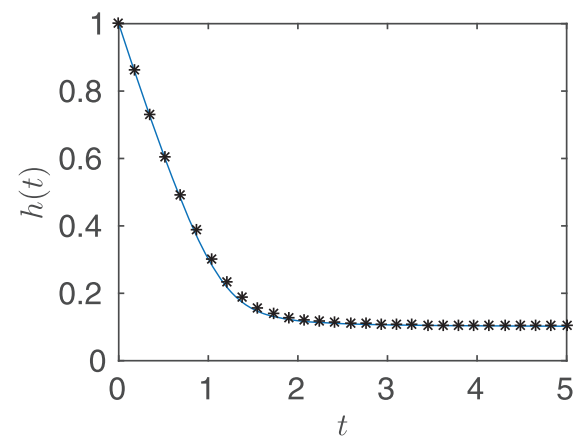

(a)

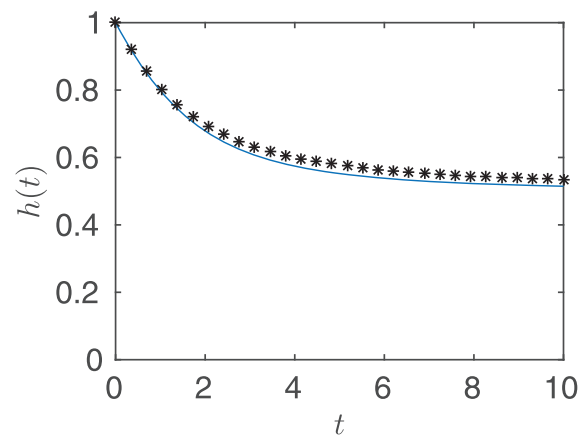

(b)

FIG. 6. Comparison of approximate (symbols) and numerical solutions (lines) for the film thickness when both drag and gravity are weak. The $R, D \ll 1$ approximation corresponds to the implicit solution in (4.3). Parameter values are: (a) $A=0.1, R=2, D=1$; (b) $A=0.5, R=0.6$, $D=0.5$. In both cases, $\mu=1$.

4.2. Strong gravity. We now assume in the nondilute model (2.10) that gravity is strong, $R \gg 1$, and that the drag coefficient $D$ is at most $O(1)$ in size. Numerical simulations of the model in this parameter regime (see Figure $7(\mathrm{a})$ ) show that the polymer (yellow) rapidly settles onto the substrate and a bilayer clearly emerges. The upper half of the bilayer is nearly pure in solvent (blue) and dries on $O(1)$ time scales.

The asymptotic analysis of the model in the strong-gravity regime is particularly difficult because the governing equations do not simplify by any appreciable amount after the appropriate scalings are made and the leading-order terms are considered. Therefore, we make use of numerical simulations to study the dynamics in this regime and briefly discuss the asymptotic structure of the solutions whenever possible.

A numerical solution of the full one-dimensional model (2.10) is shown in Figure 7. The Rayleigh number has been set to $R=100$, drag has been neglected so that $D=0$, the viscosity ratio has been set to $\mu=1$, and the mean polymer volume fraction was chosen to be $A=0.3$. Panel (a) shows the volume fraction and the clear formation of a bilayer. The profile of this bilayer at time $t=0.30$ is illustrated in panel (b). The polymer velocity and film thickness are shown in panels (c) and (d), respectively. This simulation suggests that there are three time regimes that can be considered. These are given by $t=O\left(R^{-1}\right), t \lesssim 1-A$, and $t \gtrsim 1-A$.

When $t=O\left(R^{-1}\right)$, the polymer starts settling onto the substrate and the bilayer begins to form. The asymptotic solution in this regime can be captured by scaling $t \sim R^{-1}$ and $w_{p} \sim R$. In the second time regime, $t \lesssim 1-A$, the mixture has separated into a fully developed bilayer, as shown in Figure 7(b). The spatial domain can be decomposed into outer regions near the substrate and free surfaces that are rich in polymer and solvent, respectively, and an inner region centered about $z=A$ that corresponds to a diffuse polymer-solvent interface. The governing equations for the inner region can be obtained by scaling $|z-A| \sim R^{-1}$ and $w_{p} \sim R^{-1}$, the former of which indicates that the width of the diffuse interface scales like $R^{-1}$. The momentum balance in the inner region shows that the strength of drag is characterized by the ratio $D / R^{2}$; therefore, drag is especially weak in this region. Since the upper layer of the bilayer remains dilute in polymer, it evaporates at a nearly constant and maximal rate (see section 3). Hence, the thickness of the film can be approximated by $h(t) \simeq 1-t$ for $t \lesssim 1-A$. Figure 7 (d) shows that this approximation (dashed line) is in good agreement with the numerically computed film thickness (solid line) 


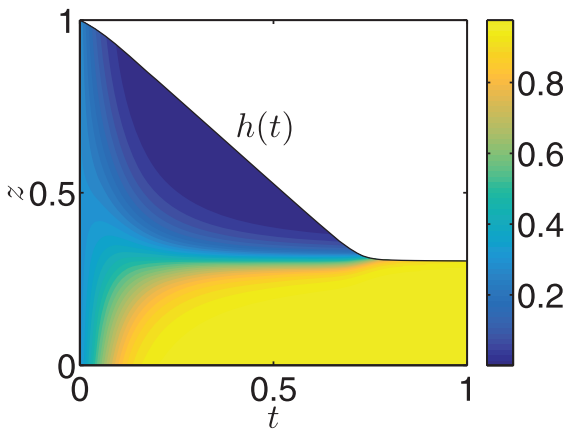

(a) $\alpha_{p}(z, t)$

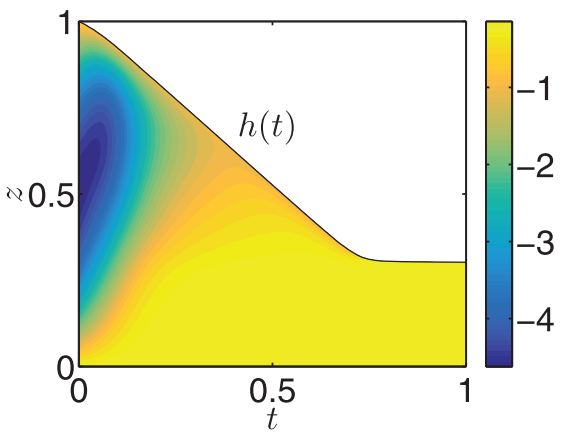

(c) $w_{p}(z, t)$

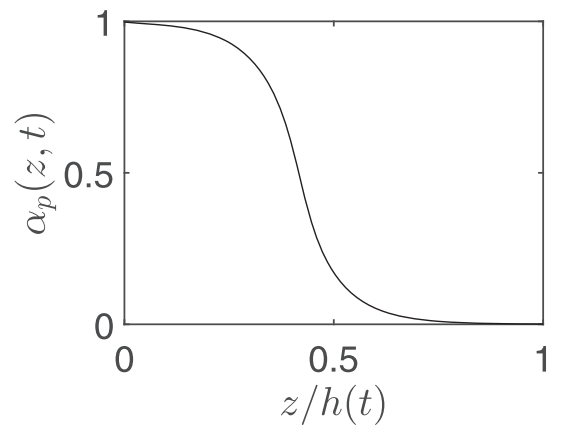

(b) $\alpha_{p}(z, t=0.30)$

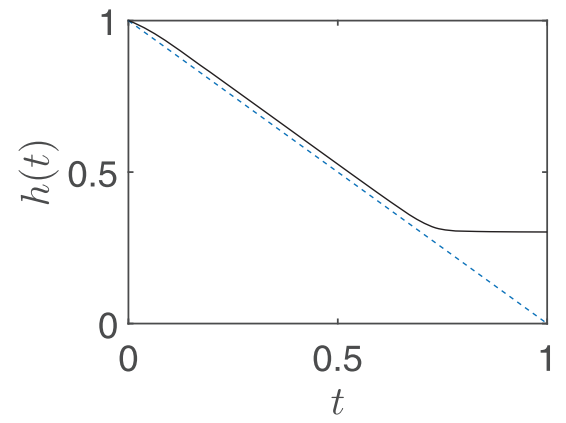

(d) $h(t)$

Fig. 7. (Color online) Dynamics when gravitational effects are strong. (a) In this case, an initially homogeneous mixture rapidly evolves into a bilayer, with the denser polymer (yellow) on the bottom and the solvent (blue) on the top. (b) Distribution of polymer in the bilayer. (c) The velocity of polymers is small near the substrate, where they are tightly packed. (d) Once the bilayer has formed, the solvent-rich upper layer freely evaporates at a constant rate. The parameters used in the simulation are $A=0.3, \mu=1, D=0, R=100$.

in this time regime. Last, the third time regime, characterized by $t \gtrsim 1-A$, captures the final stages of the drying process, namely, the removal of all remaining solvent and the formation of a pure layer of polymer.

From Figures 7(a) and (d), it can be seen that the majority of drying takes place in the second time regime, after the mixture has separated into polymer- and solvent-rich layers. The high purity of solvent-rich fluid near the free surface enables evaporation to occur at the highest rate, thus leading to the shortest possible drying times when the relative strength of gravity is large compared to drag.

4.3. Strong drag. We now consider the opposite case to that in section 4.2 by taking drag, rather than gravity, to be strong. Accordingly, we assume that $D \gg 1$ and $R=O(1)$.

An interesting situation arises when drag is strong and gravitational effects are relatively weak. On one hand, the solvent and polymer will have the tendency to travel with the same velocity due to strong drag forces. On the other hand, conservation of total mass requires that these two phases travel in opposite directions; see (2.9). The only way these two conditions can be satisfied is if both of the velocities are equal to zero. However, this cannot be the case at the free surface, where the polymer and solvent will generally have different, nonzero velocities. Thus, we expect 


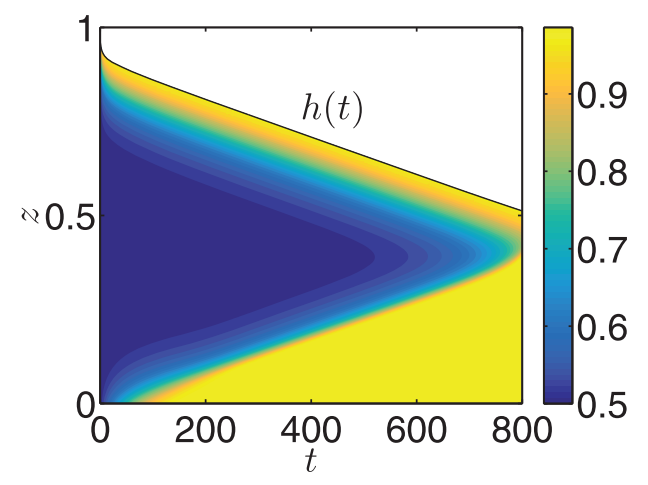

FIG. 8. (Color online) Evolution of the volume fraction $\alpha_{p}$ in the limit of strong drag, $D \gg 1$, and moderate gravity, $R=O(1)$. An asymptotic analysis reveals three time regimes. The first is when $t=O\left(D^{-1 / 2}\right)$, during which evaporation-induced drag leads to the rapid formation of a polymer-rich (yellow) skin at the free surface. When $t=O(1)$, gravitational effects lead to the slow accumulation of polymers (yellow) on the substrate. For $t \gg 1$, a two-phase core (blue) emerges and the film slowly thins until the two polymer-rich layers coalesce. Parameter values were taken to be $A=1 / 2, D=1000, R=1, \mu=1$.

the bulk of the fluid to have "outer region" behavior with a boundary layer "inner region" adjacent to the upper surface. By balancing the viscous and drag terms in the momentum balance $(2.10 \mathrm{~b})$, we estimate the width of this boundary layer to be $O\left(D^{-1 / 2}\right)$. This suggests a scaling for space that can be used when building a solution based on matched asymptotic expansions.

Numerical simulations of the nondilute model (2.10) in the strong-drag regime confirm the existence of a boundary layer near the free surface. Figure 8 shows the evolution of the volume fraction when $D=1000, R=1, A=1 / 2$, and $\mu=1$. The velocity will be discussed below. In physical terms, the formation of a boundary layer at the free surface corresponds to the growth of a polymer-rich skin. Figure 8 also indicates that moderate gravitational effects can lead to an accumulation of polymer near the substrate, similar to that observed when gravity was strong. The interior of the fluid, i.e., the region between the two polymer-rich layers, consists of a two-phase core with a composition that is uniform in both space and time. The thickness of the polymer skin remains unchanged during the drying process. The film slowly thins at a constant rate until the two polymer-rich layers coalesce (not shown).

Our analysis of this system will focus on resolving the accumulation of polymer that occurs near the free surface and the substrate. From Figure 8, we see that the development of the boundary layer near the free surface happens quickly so we introduce a fast time $\hat{t}$ defined as $\hat{t}=D^{1 / 2} t$. The power of $1 / 2$ is chosen to preserve the form of the mass balance (2.10a) in the boundary layer. On this time scale, the evolution equations for the volume fraction and film thickness become

$$
\begin{aligned}
\frac{\partial \alpha_{p}}{\partial \hat{t}}+D^{-1 / 2} \frac{\partial}{\partial z}\left(\alpha_{p} w_{p}\right) & =0, \\
\frac{\mathrm{d} h}{\mathrm{~d} \hat{t}}+D^{-1 / 2}\left[1-\alpha_{p}(h(\hat{t}), \hat{t})\right]^{2} & =0,
\end{aligned}
$$

subject to the initial conditions $\alpha_{p}(z, 0) \equiv A$ and $h(0)=1$. The hydrodynamic problem for $w_{p}$ is the same as in (2.10). 
In the outer region away from the free surface, the volume fraction and the velocity are written as $\hat{\alpha}$ and $\hat{w}$, respectively. By seeking an asymptotic expansion of the form

$$
\begin{aligned}
& \hat{\alpha}(z, \hat{t})=\hat{\alpha}_{0}+D^{-1 / 2} \hat{\alpha}_{1}+O\left(D^{-1}\right), \\
& \hat{w}(z, \hat{t})=\hat{w}_{0}+O\left(D^{-1 / 2}\right),
\end{aligned}
$$

it is straightforward to deduce that

$$
\hat{\alpha}_{0}(z, \hat{t}) \equiv A, \quad \hat{\alpha}_{1}(z, \hat{t}) \equiv 0, \quad \hat{w}_{0}(z, t) \equiv 0 .
$$

Therefore, the solution in the outer region is uninfluenced by the dynamics that occur in the inner region.

In the inner region near the free surface, the volume fraction, velocity, and film thickness are written as $\hat{P}, \hat{W}$, and $\hat{H}$, respectively. The inner problem is formulated by introducing the change of variable given by

$$
\hat{t}=\hat{t}^{\prime}, \quad \hat{\xi}=\frac{\hat{H}(\hat{t})-z}{D^{-1 / 2}} .
$$

The system of equations corresponding to (2.10a)-(2.10b) can be written as (upon dropping the primes)

$$
\begin{array}{r}
\frac{\partial \hat{P}}{\partial \hat{t}}-[1-\hat{P}(0, \hat{t})]^{2} \frac{\partial \hat{P}}{\partial \hat{\xi}}-\frac{\partial}{\partial \hat{\xi}}(\hat{P} \hat{W})=0, \\
2(1-\hat{P}) \frac{\partial}{\partial \hat{\xi}}\left(\hat{P} \frac{\partial \hat{W}}{\partial \hat{\xi}}\right)+2 \mu \hat{P} \frac{\partial}{\partial \hat{\xi}}\left[(1-\hat{P}) \frac{\partial}{\partial \hat{\xi}}\left(\frac{\hat{P} \hat{W}}{1-\hat{P}}\right)\right] \\
-\hat{P} \hat{W}-D^{-1} R \hat{P}(1-\hat{P})=0,
\end{array}
$$

which are subject to

$$
\begin{array}{cc}
\hat{P}=A, & \hat{t}=0, \\
\hat{W}=-(1-\hat{P})^{2}, & \hat{\xi}=0,
\end{array}
$$

and a matching condition for $\hat{W}$ into the outer regime that will be specified below. The film thickness evolves according to

$$
\frac{\mathrm{d} \hat{H}}{\mathrm{~d} \hat{t}}=-D^{-1 / 2}[1-\hat{P}(0, \hat{t})]^{2}
$$

with $\hat{H}(0)=1$. As before, the solution to this problem is written as an asymptotic expansion:

$$
\begin{aligned}
\hat{P}(\hat{\xi}, \hat{t}) & =\hat{P}_{0}(\hat{\xi}, \hat{t})+O\left(D^{-1 / 2}\right), \\
\hat{W}(\hat{\xi}, \hat{t}) & =\hat{W}_{0}(\hat{\xi}, \hat{t})+O\left(D^{-1 / 2}\right), \\
\hat{H}(\hat{t}) & =\hat{H}_{0}(\hat{t})+O\left(D^{-1 / 2}\right) .
\end{aligned}
$$

Copyright $@$ by SIAM. Unauthorized reproduction of this article is prohibited. 


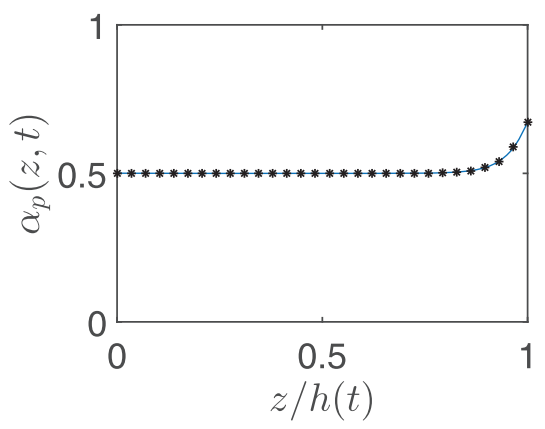

(a) $t=0.1$

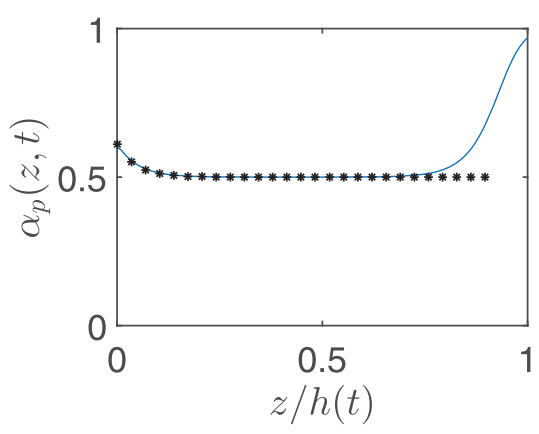

(c) $t=20$

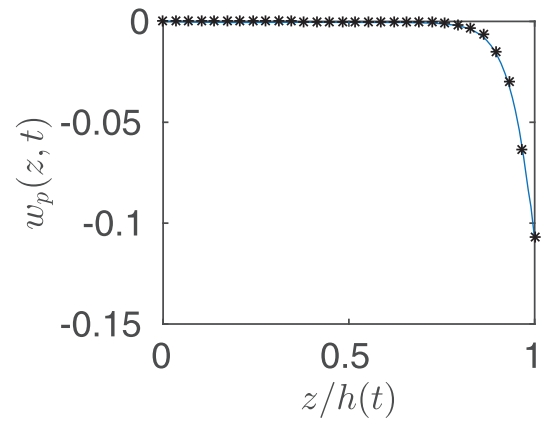

(b) $t=0.1$

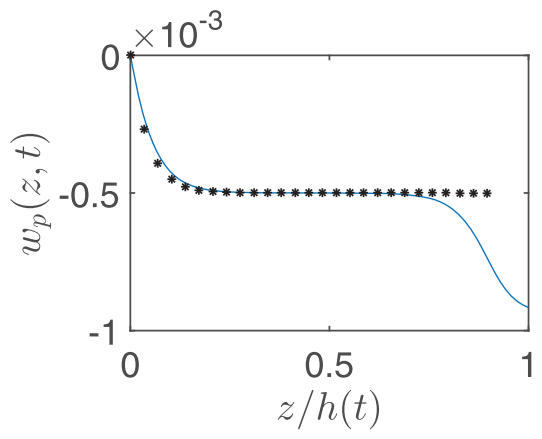

(d) $t=20$

FIG. 9. Comparisons of asymptotic solutions (symbols) to numerical solutions of the nondilute model (lines) in the limit of strong drag, $D \gg 1$, and moderate gravity, $R=O(1)$. Panels (a)-(b) depict solutions in the $t=O\left(D^{-1 / 2}\right)$ regime where a boundary layer rapidly develops at the free surface. Panels $(\mathrm{c})-(\mathrm{d})$ correspond to solutions in the $t=O(1)$ regime, where gravity leads to an accumulation of polymer near the substrate and a fully developed skin emerges at the free surface. The asymptotic solutions in this regime, given by (4.20), are valid near the substrate and in the interior of the fluid layer, hence they diverge from the numerical solutions near the free surface. Parameters values were taken to be $A=1 / 2, D=1000, R=1, \mu=1$, and they are identical to those in Figure 8.

By substituting these into (4.9e) and its initial condition, it immediately follows that

$$
\hat{H}_{0}(\hat{t}) \equiv 1 .
$$

The leading-order transport problem in this boundary layer is as complicated as the full problem; the only simplification is that gravity no longer appears. However, the leading-order problem is relatively easy to solve numerically, particularly because the computational domain no longer changes with time. Figures 9(a)-(b) show a numerical solution of the leading-order problem and compare it with a numerical solution of the full nondilute model (2.10). The agreement between the solutions is excellent. The parameters are the same as in Figure 8: $D=1000, R=1, A=1 / 2$, and $\mu=1$. The velocity is zero except in the boundary layer, where it rapidly decreases.

On longer time scales, the relatively weak, but constant, downwards pull of gravity will begin to influence the dynamics of the system, leading to the slow development of an enrichment layer near the substrate. To capture this behavior, we recast the equations in terms of the original time variable by letting $\hat{t}=D^{1 / 2} t$ with $t=O(1)$. A new velocity scale can be obtained by balancing the drag and gravity terms in the 
momentum equation (2.10b), thus giving $w_{p} \sim D^{-1}$. Therefore, in regions away from the free surface, we write $\alpha_{p}=\tilde{\alpha}$ and $w_{p}=D^{-1} \tilde{w}$, so that the corresponding problem becomes

$$
\begin{array}{r}
\frac{\partial \tilde{\alpha}}{\partial t}+D^{-1} \frac{\partial}{\partial z}(\tilde{\alpha} \tilde{w})=0 \\
2 D^{-1}(1-A \tilde{\alpha}) \frac{\partial}{\partial z}\left(\tilde{\alpha} \frac{\partial \tilde{w}}{\partial z}\right)+2 D^{-1} \mu A \tilde{\alpha} \frac{\partial}{\partial z}\left[(1-A \tilde{\alpha}) \frac{\partial}{\partial z}\left(\frac{\tilde{\alpha} \tilde{w}}{1-A \tilde{\alpha}}\right)\right] \\
-\tilde{\alpha} \tilde{w}-R \tilde{\alpha}(1-\tilde{\alpha})=0
\end{array}
$$

subject to

$$
\tilde{w}=0, \quad z=0 .
$$

The initial condition for $\tilde{\alpha}$ comes from matching into the previous time regime and we find that $\tilde{\alpha}=A+O\left(D^{-1}\right)$ as $t \searrow 0$. The solution is written as

$$
\begin{aligned}
& \tilde{\alpha}(z, t)=\tilde{\alpha}_{0}(z, t)+D^{-1 / 2} \tilde{\alpha}_{1}(z, t)+O\left(D^{-1}\right), \\
& \tilde{w}(z, t)=\tilde{w}_{0}(z, t)+O\left(D^{-1 / 2}\right) .
\end{aligned}
$$

By inserting (4.15) into (4.14), it follows that

$$
\tilde{\alpha}_{0}(z, t) \equiv A, \quad \tilde{\alpha}_{1}(z, t) \equiv 0, \quad \tilde{w}_{0}(z, t)=-R(1-A) .
$$

The solution for the vertical velocity does not satisfy the no-flux condition at the substrate (4.14c); therefore, there must be an additional boundary layer there. The solution in this layer is found by letting $z=D^{-1 / 2} \tilde{\eta}$ and writing the volume fraction and velocity as $\tilde{P}$ and $\tilde{W}$, respectively, which are found to solve

$$
\begin{aligned}
& \frac{\partial \tilde{P}}{\partial t}+D^{-1 / 2} \frac{\partial}{\partial \tilde{\eta}}(\tilde{P} \tilde{W})=0, \\
& 2(1-\tilde{P}) \frac{\partial}{\partial \tilde{\eta}}\left(\tilde{P} \frac{\partial \tilde{W}}{\partial \tilde{\eta}}\right)+2 \mu \tilde{P} \frac{\partial}{\partial \tilde{\eta}}\left[(1-\tilde{P}) \frac{\partial}{\partial \tilde{\eta}}\left(\frac{\tilde{P} \tilde{W}}{1-\tilde{P}}\right)\right] \\
& -\tilde{P} \tilde{W}-R \tilde{P}(1-\tilde{P})=0 \text {, }
\end{aligned}
$$

subject to

$$
\tilde{W}=0, \quad \tilde{\eta}=0 .
$$

The solution to this problem is written in the form

$$
\begin{aligned}
\tilde{P}(\tilde{\eta}, t) & =\tilde{P}_{0}(\tilde{\eta}, t)+D^{-1 / 2} \tilde{P}_{1}(\tilde{\eta}, t)+O\left(D^{-1}\right), \\
\tilde{W}(\tilde{\eta}, t) & =\tilde{W}_{0}(\tilde{\eta}, t)+O\left(D^{-1 / 2}\right) .
\end{aligned}
$$

The matching conditions for the $O(1)$ problem can be written as $P=A+O\left(D^{-1}\right)$ as $t \searrow 0$ and $\tilde{W}_{0} \rightarrow-R(1-A)$ as $\tilde{\eta} \rightarrow \infty$. Analytical solutions to the inner problem near the substrate can be obtained and combined with the outer solutions to form pseudocomposite solutions given by

$$
\begin{aligned}
& \alpha_{p}(z, t)=A+\frac{A R t}{\lambda D^{1 / 2}} \exp \left(-D^{1 / 2} z / \lambda\right) \\
& w_{p}(z, t)=-D^{-1} R(1-A)\left[1-\exp \left(-D^{1 / 2} z / \lambda\right)\right],
\end{aligned}
$$

Copyright $@$ by SIAM. Unauthorized reproduction of this article is prohibited. 


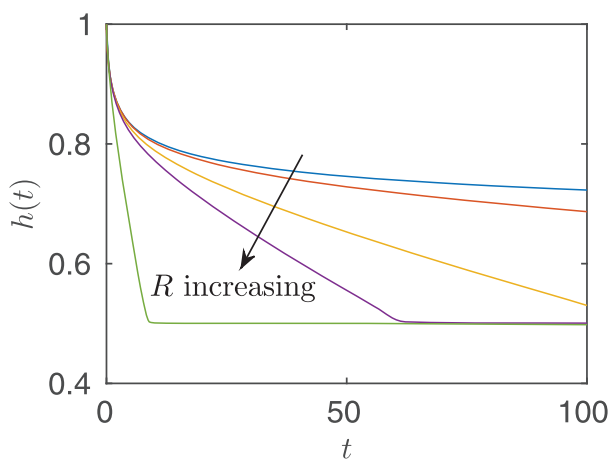

FIG. 10. Evolution of the film thickness when $D \gg 1$ for different values of the Rayleigh number $R$. There is a strong dependence of the drying time on $R$ in this parameter regime. Parameter values are $A=1 / 2, \mu=1, D=100$, and $R=0,0.1,0.5,1$, and 10 .

where $\lambda$ is a constant defined as

$$
\lambda=\{2[1+(\mu-1) A]\}^{1 / 2} .
$$

The expressions in (4.20) are called pseudocomposite solutions because they do not satisfy the boundary conditions at the free surface. In fact, the continued growth of the boundary layer at the free surface in this time regime leads to the formation of a fully developed polymer-rich skin.

Panels (c) and (d) of Figure 9 compare the pseudocomposite asymptotic solutions in the $t=O(1)$ regime given by (4.20) to numerical simulations of the nondilute model (2.10). Again, the agreement between the two are excellent in the region of validity. The characteristic features of the solution in this time regime are the emergence of a boundary layer at the substrate caused by compositional buoyancy and a polymer-rich skin at the free surface. Unlike in the early-time regime, the velocity in the interior of the mixture is now nonzero and can be determined by balancing drag and buoyancy terms in the momentum equation (2.10).

The relative strength of gravity plays a primary role in setting the drying time of the film when drag is strong. Stronger gravity reduces the volume fraction of polymer below the free surface and inhibits skin formation, therefore resulting in higher rates of evaporation. This behavior is clearly illustrated by Figure 10, which shows the evolution of the film thickness when $D \gg 1$ for several different values of $R$.

4.4. Strong drag and strong gravity. The qualitative features of the system in the case of strong drag, $D \gg 1$, and strong gravity, $R \gg 1$, are similar to those seen previously. In particular, gravity leads to the rapid development of an enrichment layer near the substrate, and drag quickly results in the formation of a polymerrich skin. The dominant layer that emerges in the early stages of evolution can be determined by carrying out a small-time analysis of the nondilute model (2.10) assuming that $R \gg 1$ and $D \gg 1$, with no restriction on the relative sizes of these parameters. By letting $t=\varepsilon \tau$, where $\varepsilon \ll 1$ is sufficiently small, and expanding the solution in powers of $\varepsilon$, it is straightforward to show that the greatest accumulation of polymer will be on the substrate when $R>(1-A) D / 2$ and near the free surface when $R<(1-A) D / 2$. These inequalities, in fact, hold regardless of the sizes of $R$ and $D$, and they generalize the gravity- and drag-dominated regimes that were computed in section 3 to nondilute polymer concentrations. 
5. Discussion and conclusion. In this paper, we formulated a two-phase model for an evaporating solvent-polymer mixture and used it to explore the interplay between gravity, evaporation, and drag. Under the assumption that the polymer was denser than the solvent, we found that gravitational effects drove an accumulation of polymer on top of the substrate. Drag and evaporation were responsible for the formation of a polymer-rich skin below the free surface. A small-time analysis of the model produced an explicit condition for assessing which of these two accumulations of polymer will be the greatest. Particular attention was paid to determining the drying time of the mixture, since this is often a quantity of practical interest. When gravity is the dominant mechanism driving the flow, the system quickly settles into a bilayer configuration and the solvent-rich upper layer evaporates at an optimal rate, leading to the shortest drying times. When drag is dominant, the skin inhibits the evaporation of solvent, significantly increasing the drying time. However, moderate increases to the relative strength of gravity were found to substantially decrease the drying time in the strong-drag regime, suggesting a possible way to mediate skin formation or its effects is through the use of a low-density solvent, for example.

The density stratification that arises from the depletion of solvent near the free surface leads to the question of whether the system is susceptible to a buoyancy-driven instability. The onset of such an instability is likely to have important practical consequences, as it could induce the break up of a skin and influence the morphology of the dried polymer film. Although we did not explore the possibility of an instability here, our analysis does give some insight into the conditions under which this might occur. For an instability driven by compositional buoyancy to be relevant, it must develop before the film dries. This is expected to be the case when drag is strong. If gravity is strong but drag is weak, the rapid settling of the polymer onto the substrate will prevent an unstable density stratification. Using our model, the role of compositional buoyancy in the evaporating polystyrene-toluene blends studied by Bassou and Rharbi [1] can be estimated. They report that $\rho_{p}-\rho_{s} \sim 10^{2} \mathrm{~kg} / \mathrm{m}^{3}, E \sim 10^{-7} \mathrm{~m} / \mathrm{s}$, and $H \sim 10^{-3} \mathrm{~m}$, leading to a Rayleigh number of $R \sim\left(10^{4} \mathrm{~Pa} \mathrm{~s}\right) / \mu_{p}$. Unless $\mu_{p}$ is large, the effects of compositional buoyancy will be significant in these experiments, a conclusion that was also reached by Hennessy and Münch [18]. Therefore, buoyancy could act in conjunction with other mechanisms, such as surface-tension gradients, to drive convection within these fluid layers.

In reality, there will be several physical mechanisms that mediate the effects of a skin. In addition to gravity, diffusion of polymer and solvent can play an important role. For example, diffusion of solvent through the skin may provide a mechanism by which evaporation can continue, albeit at a very slow rate. Studies of spin-coated solvent-polymer mixtures by Münch, Please, and Wagner [28] confirm the close link between evaporation, diffusion, and skin formation. In particular, they find that when evaporation is sufficiently slow, diffusion can flatten the concentration profile, thus enabling all of the solvent to be removed from the layer. Qualitatively similar dynamics are observed in the two-phase model when both gravity and drag are weak.

The simplicity of our two-phase fluid model prevents it from capturing physical effects that may play important roles during the drying process. For instance, many polymeric fluids exhibit non-Newtonian [24] or viscoelastic $[4,16]$ rheologies and, in fact, can turn into brittle solids if the glass transition is crossed [8]. Such effects, while likely negligible during the early stages of film drying due to the low initial concentration of polymer, can become important during the later stages where, in particular, they will influence the development and evolution of the polymer-rich skin. Although our model describes a highly idealized solvent-polymer mixture, it 
benefits from additional mathematical tractability, enabling further insights to be made into the relationship between evaporation, gravity, and skin formation. In this paper, we have highlighted how even a relatively simple two-phase model can have a rich asymptotic structure and wide range of interesting dynamics. We hope this work will stimulate future investigations focusing on the development and asymptotic analysis of multiphase models for evaporating solvent-polymer mixtures.

Appendix A. Derivation of a drift-flux model. The drift-flux models that are often used to describe evaporating solvent-polymer mixtures can be derived from the two-phase model (2.1) by simplifying the mass and stress balances for the mixture, (2.1b) and (2.1d), and replacing (2.1c) with a phenomenological equation that describes the flow of polymer. The derivation begins by introducing the mean mixture velocity and viscous stress,

$$
\begin{aligned}
w & =\left(1-\alpha_{p}\right) w_{s}+\alpha_{p} w_{p}, \\
\sigma & =\left(1-\alpha_{p}\right) \sigma_{s}+\alpha_{p} \sigma_{p},
\end{aligned}
$$

respectively. For the model that is presented in (2.1), we have taken $\sigma_{k}=2 \mu_{k} \partial w_{k} / \partial z$, $k=s, p$, but this is immaterial here. From (2.1b) and (2.1d), the mass and momentum balances for the mixture can be written as

$$
\begin{aligned}
\frac{\partial w}{\partial z} & =0 \\
-\frac{\partial p}{\partial z}+\frac{\partial \sigma}{\partial z}-\left[\left(1-\alpha_{p}\right) \rho_{s}+\alpha_{p} \rho_{p}\right] g & =0 .
\end{aligned}
$$

Often, the mixture stress is assumed to be of the form

$$
\sigma=2 \mu_{m}\left(\alpha_{p}\right) \frac{\partial w}{\partial z}
$$

or a multidimensional equivalent, where $\mu_{m}$ is the composition-dependent mean viscosity of the mixture. The flux of polymer is assumed to consist of an advective flux associated with the mixture velocity and a Fickian diffusive flux,

$$
\alpha_{p} w_{p}=\alpha_{p} w-\mathcal{D}\left(\alpha_{p}\right) \frac{\partial \alpha_{p}}{\partial z},
$$

where $\mathcal{D}$ is the mutual diffusion coefficient of solvent and polymer. The relationship in (A.4) is used in place of the momentum balance (2.1c). Furthermore, an advectiondiffusion equation for the polymer volume fraction can be obtained by inserting (A.4) into (2.1a) and using (A.2a):

$$
\frac{\partial \alpha_{p}}{\partial t}+w \frac{\partial \alpha_{p}}{\partial z}=\frac{\partial}{\partial z}\left(\mathcal{D}\left(\alpha_{p}\right) \frac{\partial \alpha_{p}}{\partial z}\right) .
$$

The system of equations given by (A.2), (A.3), and (A.5) forms the drift-flux model of a solvent-polymer blend. The drift-flux model cannot be obtained through a systematic reduction of the two-phase model (2.1) because the latter does not include a physical mechanism that leads to diffusive behavior. However, extensions of the twophase model that consider the osmotic pressure can introduce diffusive terms [36], making it possible to recover the drift-flux and well-mixed models.

Appendix B. Details of the numerical method. In principle, the two-phase model can be studied via numerical simulations of (2.10). However, we found that 
this system is particularly prone to numerical instabilities, especially when singlephase regions, corresponding to $\alpha_{p} \simeq 0$ or $\alpha_{p} \simeq 1$, begin to appear. These difficulties prevent the numerical method from capturing key asymptotic regimes predicted by our analysis. Therefore, we opt to solve an intermediate system that lies somewhere between the original model (2.3)-(2.8) and the reduced model (2.10).

We start by integrating the incompressibility condition (2.3b) and applying the no-flow conditions at $z=0$ to obtain

$$
\left(1-\alpha_{p}\right) w_{s}+\alpha_{p} w_{p}=0 .
$$

We do not use (B.1) to eliminate one of the fluid velocities, as this would introduce singularities in the system when $\alpha_{p} \rightarrow 0$ or $\alpha_{p} \rightarrow 1$. The momentum balance for the mixture (2.3d) is used to obtain an expression for the pressure gradient, which is then inserted into (2.3c) to obtain

$$
\begin{aligned}
2\left(1-\alpha_{p}\right) \frac{\partial}{\partial z}\left(\alpha_{p} \frac{\partial w_{p}}{\partial z}\right) & -2 \mu \alpha_{p} \frac{\partial}{\partial z}\left[\left(1-\alpha_{p}\right) \frac{\partial w_{s}}{\partial z}\right] \\
& +D \alpha_{p}\left(1-\alpha_{p}\right)\left(w_{s}-w_{p}\right)=R \alpha_{p}\left(1-\alpha_{p}\right) .
\end{aligned}
$$

We supplement (B.2) with the boundary conditions given by

$$
\begin{array}{ll}
w_{p}=0, & z=0, \\
w_{p}=-\left(1-\alpha_{p}\right)^{2}, & z=h(t) .
\end{array}
$$

Boundary conditions for the solvent velocity do not need to be explicitly imposed because the integrated incompressibility condition (B.1) ensures that they will be satisfied. This can be verified by evaluating (B.1) on the boundaries and using (B.3).

The system given by (B.1)-(B.3) constitutes the hydrodynamic problem that is solved in our numerical method. The advantage of this system over (2.10b) is that it remains well defined as $\alpha_{p} \simeq 0$ and $\alpha_{p} \simeq 1$; note that (2.10b) becomes singular as $\alpha_{p} \rightarrow 1$, for example.

Rather than solving a nonlinear system at each time step, we decouple the transport and hydrodynamic problems. Given the polymer volume fraction and film thickness at time $t$, we solve the linear hydrodynamic problem (B.1)-(B.3) to obtain the solvent and polymer velocities. The newly computed polymer velocity is then used in the time-stepping scheme to update the volume fraction and the film thickness, and the procedure then repeats.

To deal with the free boundary, we use the change of variable $\eta=z / h(t)$ to map the problem onto a fixed domain. We then discretize spatial derivatives using finitedifference formulas. Upwinding is used when discretizing the conservation equation (2.10a). The MATLAB ode45 function is used for time stepping.

Acknowledgments. MH wishes to thank Tobias Ahnert, Jon Chapman, and Peter Howell for many stimulating discussions about multiphase flows.

\section{REFERENCES}

[1] N. Bassou And Y. Rharbi, Role of Bénard-Marangoni instabilities during solvent evaporation in polymer surface corrugations, Langmuir, 25 (2009), pp. 624-632.

[2] G. W. Bergantz And J. Ni, A numerical study of sedimentation by dripping instabilities in viscous fluids, Int. J. Multiph. Flow, 25 (1999), pp. 307-320. 
[3] D. E. Bornside, C. W. Macosko, And L. E. Scriven, Spin coating: One-dimensional model, J. Appl. Phys., 66 (1989), pp. 5185-5193.

[4] R. A. Cairncross and C. J. Durning, A model for drying of viscoelastic polymer coatings, AIChE J., 42 (1996), pp. 2415-2425.

[5] S. Cataldo, C. Sartorio, F. Giannazzo, A. Scandurra, and B. Pignataro, Selforganization and nanostructural control in thin film heterojunctions, Nanoscale, 6 (2014), pp. 3566-3575.

[6] L.-M. Chen, Z. Hong, G. LI, AND Y. YAnG, Recent progress in polymer solar cells: Manipulation of polymer: Fullerene morphology and the formation of efficient inverted polymer solar cells, Adv. Mater., 21 (2009), pp. 1434-1449.

[7] B.-J. De Gans, P. C. Duineveld, and U. S. Schubert, Inkjet printing of polymers: State of the art and future developments, Adv. Mater., 16 (2004), pp. 203-213.

[8] P.-G. DE Gennes, Solvent evaporation of spin cast films: "Crust" effects, Eur. Phys. J. E, 7 (2002), pp. 31-34.

[9] F. Doumenc, T. Boeck, B. Guerrier, And M. Rossi, Transient Rayleigh-Bénard-Marangoni convection due to evaporation: A linear non-normal stability analysis, J. Fluid Mech., 648 (2010), pp. 521-539.

[10] D. A. Drew, Mathematical modeling of two-phase flow, Annu. Rev. Fluid Mech., 15 (1983), pp. 261-291.

[11] D. A. Drew and S. L. Passman, Theory of Multicomponent Fluids, Springer, New York, 1999.

[12] A. D. F. Dunbar, P. Mokarian-Tabari, A. J. Parnell, S. J. Martin, M. W. A. Skoda, AND R. A. L. JonEs, A solution concentration dependent transition from self-stratification to lateral phase separation in spin-cast PS:d-PMMA thin films, Eur. Phys. J. E, 31 (2010), pp. 369-375.

[13] S. Ebbens, R. Hodgkinson, A. J. Parnell, A. Dunbar, S. J. Martin, P. D. Topham, N. Clark, AND J. R. Howse, In situ imaging and height reconstruction of phase separation processes in polymer blends during spin coating, ACS Nano, 5 (2011), pp. 5124-5131.

[14] M. H. Eres, D. E. Weidner, And L. Schwartz, Three-dimensional direct numerical simulation of surface-tension-gradient effects on the leveling of an evaporating multicomponent fluid, Langmuir, 15 (1999), pp. 1859-1871.

[15] P. L. Evans, L. W. Schwartz, and R. V. Roy, A mathematical model for crater defect formation in a drying paint layer, J. Colloid Interface Sci., 227 (2000), pp. 191-205.

[16] G. Harrison, J. LAmb, AND A. J. Matheson, The viscoelastic properties of dilute solutions of polystyrene in toluene, J. Phys. Chem., 68 (1964), pp. 1072-1078.

[17] M. G. Hennessy, Mathematical Problems Relating to the Fabrication of Organic Photovoltaic Devices, Ph.D. thesis, University of Oxford, Oxford, England, 2014, http://ora.ox.ac.uk/ objects/uuid:7753abec-bb6e-4d8a-aa5b-b527c5beb49b.

[18] M. G. Hennessy And A. MÜnCH, A multiple-scale analysis of evaporation induced Marangoni convection, SIAM J. Appl. Math., 73 (2013), pp. 974-1001.

[19] M. G. Hennessy AND A. MÜNCH, Dynamics of a slowly evaporating solvent-polymer mixture with a deformable upper surface, IMA J. Appl. Math., 79 (2014), pp. 681-720.

[20] S. Y. Heriot And R. A. L. Jones, An interfacial instability in a transient wetting layer leads to lateral phase separation in thin spin-cast polymer-blend films, Nature Mater., 4 (2005), pp. $782-786$.

[21] S. D. Howison, J. A. Moriarty, J. R. Ockendon, E. L. Terrill, and S. K. Wilson, A mathematical model for drying paint layers, J. Engrg. Math., 32 (1997), pp. 377-394.

[22] M. Ishi And T. HiBiki, Thermo-Fluid Dynamics of Two-Phase Flow, 2nd ed., Springer, New York, 2011.

[23] J. Jaczewska, A. Budkowski, A. Bernasik, E. Moons, and J. Rysz, Polymer vs. solvent diagram of film structures formed in spin-cast poly(3-alkylthiophene) blends, Macromolecules, 41 (2008), pp. 4802-4810.

[24] W.-M. Kulicke And R. Kniewske, The shear viscosity dependence on concentration, molecular weight, and shear rate of polystyrene solutions, Rheol. Acta, 23 (1984), pp. 75-83.

[25] H. Machrafi, A. Rednikov, P. Colinet, and P. C. Dauby, Bénard instabilities in a binaryliquid layer evaporating into an inert gas, J. Colloid Interface Sci., 349 (2010), pp. 331-353.

[26] P. Mokarian-Tabari, M. Geoghegan, J. R. Howse, S. Y. Heriot, R. L. Thompson, and R. A. L. JONES, Quantitative evaluations of evaporation rate during spin-coating of polymer blend films: Control of film structure through defined-atmosphere solvent-casting, Eur. Phys. J. E, 33 (2010), pp. 283-289.

[27] E. Moons, Conjugated polymer blends: Linking film morphology to performance of light emitting diodes and photodiodes, J. Phys. Condensed Matter, 14 (2002), pp. 12235-12260.

[28] A. Münch, C. P. Please, And B. Wagner, Spin coating of an evaporating polymer solution, Phys. Fluids, 23 (2011), 102101.

Copyright (c) by SIAM. Unauthorized reproduction of this article is prohibited. 
[29] C. Park, J. Yoon, And E. L. Thomas, Enabling nanotechnology with self assembled block copolymer patterns, Polymer, 44 (2003), pp. 6725-6760.

[30] L. Pauchard and C. Allain, Buckling instability induced by polymer solution drying, Europhys. Lett., 62 (2003), pp. 897-903.

[31] B. Reisfeld, S. G. Bankoff, And S. H. Davis, The dynamics and stability of thin liquid films during spin coating. II. Films with unit-order and large Peclet numbers, J. Appl. Phys., 70 (1991), pp. 5267-5277.

[32] S. Walheim, M. Böltau, J. Mlynek, G. Krausch, and U. Steiner, Structure formation via polymer demixing in spin-cast films, Macromolecules, 30 (1997), pp. 4995-5003.

[33] S. Walheim, E. Schäffer, J. Mlynek, and U. Steiner, Nanophase-separated polymer films as high-performance antireflection coatings, Science, 283 (1999), pp. 520-522.

[34] D. E. Weidner, L. W. Schwartz, and R. R. Eley, Role of surface tension gradients in correcting coating defects in corners, J. Colloid Interface Sci., 179 (1996), pp. 66-75.

[35] S. K. Wilson, The levelling of paint films, IMA J. Appl. Math., 50 (1993), pp. 149-166.

[36] H. F. Winstanley, M. Chapwanya, M. J. McGuinness, and A. Fowler, A polymer-solvent model of biofilm growth, R. Soc. Proc. A Math. Phys. Eng. Sci., 467 (2010), pp. 1449-1467.

Copyright (c) by SIAM. Unauthorized reproduction of this article is prohibited. 